\title{
La gestión informativa del riesgo medioambiental
}

\author{
José Ignacio LORENTE* \\ eneko.lorente@ehu.es
}

(Abstracts y palabras clave al final del artículo)

Enviado: 20 de abril de 2013

Evaluado: 2 de julio de 2013

Aceptado: 12 de julio de 2013

\section{INTRODUCCIÓN}

Hasta el estallido de la actual crisis económica, la inquietud por el riesgo medioambiental ha sido objeto de atención pública creciente a lo largo de los últimos años. Sin embargo, en el umbral de la crisis, diversos estudios (Lowe, 2006; Imbert, 2008; Beck, 2010; Norgaard, 2011) subrayaban la paradoja de una ciudadanía que, pese a manifestar preocupación por los impactos en el medio ambiente, se mostraba escéptica ante la capacidad de participación en las diferentes dimensiones que comporta su mitigación. Esos mismos estudios advertían también del enfoque catastrofista con que los medios de comunicación abordaban los riesgos medioambientales y de los consiguientes efectos de falta de implicación y escepticismo en la ciudadanía.

Este trabajo trata de analizar la posible relación existente entre el tratamiento informativo que la prensa diaria proporciona a la problemática medioambiental y el escepticismo que la ciudadanía manifiesta acerca de su capacidad para participar en su gestión. Para ello, el objetivo de este análisis consiste en profundizar en el conocimiento del discurso del riesgo presente en las noticias publicadas en la prensa española de mayor difusión (El País, El Mundo, La Vanguardia y ABC), en relación con la política del cambio climático, en el marco temporal de las conferencias internacionales auspiciadas por Naciones Unidas (UNFCCC) a lo largo de los últimos cuatro años, con motivo de las negociaciones para la renovación del protocolo de Kioto (2009-12): COP 15 (Copenhague, 7-18/XII/2009), COP 16 (Cancún, 29/1110/XII/2010), COP 17 (Durban, 28/11-9/XII/2011) y COP 18 (Doha, 26/118/XII/2012). De la información publicada en esas fechas se ha seleccionado un corpus de 54 piezas informativas, tomando como criterio tres secuencias características

\footnotetext{
* Profesor Titular de Universidad de la Universidad del País Vasco (UPV/EHU), en el Departamento de Comunicación Audiovisual y Publicidad de la Facultad de Ciencias Sociales y de la Comunicación.
} 
del relato de los conflictos suscitados en torno a la ratificación de acuerdos para el afrontamiento de los efectos del cambio climático. Estas secuencias reiteradas en el seguimiento informativo de cada conferencia, responden a una dramaturgia característica que se inicia con la identificación de objetivos, creación de expectativas y anticipación de los contextos de negociación (16 piezas, 29,62\% de la muestra), continua con el tratamiento de las principales controversias y obstáculos para el acuerdo (22 piezas, 40,74\% de la muestra); y finaliza con la valoración de logros y fracasos, referida tanto a los resultados de las negociaciones como a la satisfacción de las expectativas iniciales (16 piezas, $29,63 \%$ de la muestra analizada).

Una de las cuestiones más relevantes del seguimiento informativo de esas conferencias consiste en que, si bien fueron concebidas como un foro político de discusión con amplia visibilidad pública, los medios de comunicación han presentado la deliberación política como un obstáculo para la adopción de estrategias de afrontamiento de los inminentes riesgos medioambientales. Este sesgo informativo resulta significativo en el seguimiento de la cumbre de Copenhague (2009), donde las expectativas de renovación del protocolo de Kioto fueron frustradas por la resistencia de los principales productores de gases de efecto invernadero a asumir compromisos verificables de reducción, así como las sanciones correspondientes en caso de incumplimiento.

Por otra parte, la confianza expresada desde la Conferencia de Bali (2007) en el consenso científico acerca del carácter antropogénico de los impactos ambientales, parecía haber creado la ilusión de que ese mismo consenso podría imponerse como criterio para el acuerdo en materia política, trasladando la lógica científica a la lógica social. Sin embargo, esta traslación obviaba el hecho de que el objeto de ambos consensos es diferente. Así, en tanto que el primero consiste en la determinación de las causas y alcance del deterioro medioambiental, la falta de acuerdo político se refiere al disenso en la definición del riesgo (Lorenzoni y Pidgeon, 2006; Slimak y Dietz, 2006; Pettenger, 2007; Boykoff, 2011) y al reparto desigual de los impactos previstos.

La hipótesis de partida frente a estos sesgos informativos se basa en el supuesto de que el tratamiento dramatizado y reiteradamente catastrofista con que la prensa de actualidad aborda los problemas y conflictos medioambientales pudiera favorecer la falta de expectativas de la ciudadanía acerca de la pertinencia de acuerdos políticos multilaterales para afrontar los principales riesgos. El trabajo indaga también la presencia de narrativas escalares que, en función del ámbito espacial de referencia, perfilan escenarios diferenciados de acción política y prefiguran la competencia de los sujetos llamados a ejercerla. En este sentido, cabe destacar que los principales productores de factores de riesgo medioambiental son precisamente aquellos con mayor capacidad para determinar su alcance, para valorarlos en términos de coste-beneficio y para actuar sobre los mismos desde la lógica de la racionalidad técnica y la oportunidad.

Finalmente, se trata de concluir de todo ello cómo la participación de la ciudadanía en la mitigación y eventual adaptación a los problemas medioambientales no descansa únicamente en la disponibilidad de información y en el conocimiento acerca de los mismos. Participar requiere además la atribución de sentido a los cursos de 
acción a través de la deliberación y toma de decisiones acerca de estrategias consensuadas para su afrontamiento, en el ámbito de actuación ciudadana.

Por esta razón, si bien el tratamiento informativo de los problemas medioambientales ha contribuido a la divulgación del conocimiento del consenso científico (Díaz Nosty, 2009), lo ha hecho proyectando dicho conocimiento sobre escenarios sociales que no se guían únicamente en términos de racionalidad científica. La información de actualidad al abordar los riesgos medioambientales en términos de globalidad, inminencia e inexorabilidad de la crisis, ha contribuido también a evacuar su carácter eminentemente político, obviando las diferentes atribuciones de valor y el interés con que socialmente es definido el objeto del riesgo. En este sentido, el tratamiento catastrofista del riesgo medioambiental, al igual que ocurre con los riesgos sanitarios o financieros, contribuye eficazmente a la gestión de los mismos, si bien lo hace a costa de promocionar la urgencia de medidas sujetas a la razón técnica, en detrimento de la deliberación política.

A este respecto, resulta significativo que, si bien diversos estudios de opinión relacionados con la problemática medioambiental, como el Flash Eurobarometer (2010/290) o el Special Eurobarometer on Climate Change (2011/372) indicaban un aumento de la preocupación de los ciudadanos europeos con respecto al cambio climático, por delante incluso de la crisis económica, se mostraban sin embargo confiados en las soluciones tecno-científicas y en la posibilidad de que las medidas para combatirlo pudieran beneficiar a la economía y al empleo. En el caso de España, pese a que los ciudadanos sitúan los problemas medioambientales entre los menos valorados de los problemas que afectan al conjunto del país (CIS, 2011/2909; 2012/2972), muy alejados del paro y de la crisis económica, otro estudio más específico (CIS, International Social Survey Programme, 2837/2010) ponía de manifiesto que, cuando son preguntados por los temas relativos al medio ambiente, dos de cada tres ciudadanos afirman estar preocupados o muy preocupados por los mismos, señalando el cambio climático como el principal problema medioambiental. Sin embargo, pese a que los ciudadanos se sienten directamente afectados por estos problemas y afirman disponer de información suficiente, uno de cada tres manifiesta desconocer sus causas y casi la mitad ignora las posibles soluciones objeto de discusión. La misma proporción considera que, de una u otra forma, la ciencia solucionará los problemas medioambientales, sin que se produzcan grandes cambios en el estilo de vida actual.

Estas apreciaciones, con todas las reservas precisas, ponen de relieve que si bien la opinión pública se halla más sensibilizada que en el pasado acerca de la crisis de sostenibilidad que afecta al modelo productivo y los estilos de vida, su relación con los problemas medioambientales se percibe de forma difusa e indeterminada. Por otra parte, las expectativas puestas en el conocimiento y en el progreso tecno-científico para asegurar la continuidad del modelo de crecimiento hasta ahora practicado, una vez superada la crisis, dibujan un panorama de desmovilización y de descrédito de una parte significativa de la ciudadanía hacia la propia capacidad e iniciativa para la gestión y paliación de los principales problemas medioambientales.

En este contexto de desmovilización, los medios informativos parecen haber tenido un papel relevante tanto en lo referente a la comunicación del acontecer 
medioambiental, como en su papel de divulgadores de la creencia de que quien está capacitado para definir y evaluar técnicamente el riesgo, será capaz también de poner las medidas técnicas necesarias para afrontarlo, independientemente de la deliberación política.

La problemática medioambiental entró a formar parte de la agenda pública a partir del momento en que Naciones Unidas, basándose en los estudios del Panel Intergubernamental sobre Cambio Climático (IPCC) y los posibles escenarios resultantes del calentamiento del sistema climático, llamara la atención acerca de la necesidad de que tanto los gobiernos, instituciones y organizaciones, como la propia ciudadanía se involucrasen en el desarrollo de programas de actuación frente a los principales riesgos medioambientales. El IPCC esbozó complejos escenarios del calentamiento en los que se combinaban múltiples factores, tales como el crecimiento económico, el agotamiento de los recursos naturales, el incremento demográfico o el acrecentamiento de las desigualdades regionales. Pero, si bien en ninguno de esos escenarios era previsible el fin del planeta, advierten sus autores que, en cualquiera de estas proyecciones, los conflictos por el acceso a los recursos naturales y por el reparto desigual de los impactos ambientales afectarán tanto a la geopolítica y a las relaciones internacionales, como a la desigualdad y a la cohesión social (Rajendra, 2007: 61).

En términos generales, los medios de comunicación han mantenido esa preocupación en la agenda informativa referida tanto a los acontecimientos relacionados con los escenarios de riesgo, como a los encuentros internacionales donde se negocian y dirimen las estrategias destinadas a su afrontamiento. Sin embargo, si bien el avance del conocimiento científico en este campo ha sido objeto de la atención informativa, su tratamiento ha puesto frecuentemente el énfasis en el peor de los escenarios posibles y en la dramatización del disenso como lógica narrativa privilegiada.

Los titulares con los que la prensa española ha relatado las negociaciones en las conferencias sobre el cambio climático resultan significativos en la medida en que ponen en escena, bajo el argumento de la urgencia de medidas para afrontar los problemas medioambientales, la dramática necesidad de evacuar la deliberación y el disenso en torno a las mismas: "La negociación del clima, un zombi" (Sabartés, $L a$ Vanguardia, 11/XII/2010), "Torre-cumbre de Babel en Copenhague" (Javaloyes, $A B C, 19 / 12 / 2009)$, "La ONU sepulta la esperanza" (Méndez, El País, 7/XII/2011), "La Cumbre del Clima de Doha llega a su recta final bajo la sombra del fracaso" (Agencias, El Mundo, 4/XII/2012), "La negociación del clima encalla en Doha" (Méndez, El País, 8/XII/2012).

Sin embargo, el disenso acerca de la forma de afrontar los escenarios posibles no significa inacción, sino que expresa más bien las diferentes posiciones, intereses y valores en juego en la definición, selección y estrategias de acción frente al riesgo medioambiental. En la medida en que no existe una naturaleza ajena a lo social, la definición del riesgo no trata sólo de las probabilidades de que cualquiera de los escenarios posibles pueda llegar a realizarse, sino de los umbrales de afectación que una sociedad está dispuesta a tolerar y de aquellos aspectos de la naturaleza y de las formas privilegiadas de producción y de consumo de las que está dispuesta a prescindir. De la movilidad de estos umbrales dan cuenta los discursos del riesgo y las narrativas informativas desde las que se gestionan los conflictos que llevan asociados. 


\section{EL DISCURSO DEL RIESGO MEDIOAMBIENTAL}

Este trabajo se inscribe en el campo de una teoría del discurso que observa en este cierta disposición estratégica destinada a poner en movimiento la significación, la cual lejos de representar meros estados del mundo busca transformar dichos estados, modificando al mismo tiempo a quien lo produce y a quien lo interpreta y comprende.

La nueva perspectiva sobre el discurso resultante del giro textual advierte en él un carácter polémico, un campo de tácticas y de maniobras, cuya finalidad no se resuelve en una hipotética transmisión de información, sino que implica además la definición y manipulación de la competencia interpretativa de los sujetos llamados a participar en la interacción comunicativa (Fabbri, 2004: 47). El reconocimiento o refutación de esa competencia constituye un modelo de acción que el texto dispone y gestiona en términos pragmáticos y que tiene su correlato en las prácticas sociales, allí donde ese sujeto textualmente construido se reconoce competente para participar en determinados cursos de acción social y política (Rancière, 2006: 56).

El método de análisis se ha centrado en la identificación de los marcos espaciotemporales (framing) en los que el discurso del riesgo inscribe los acontecimientos y problemas medioambientales y en el reconocimiento de la primacía de ciertos actores para actuar en ellos, en detrimento de otros posibles participantes. Esta perspectiva metodológica asume que "los diarios no se limitan a reproducir hechos ya ocurridos, sino que tratan de producir acontecimientos" (Fabbri, 1995: 311), movilizando el sentido de los mismos a través de la narratividad, de la capacidad configurante del relato (Ricoeur, 2008: 482). Los relatos informativos combinan de forma específica "no sólo palabras, frases o proposiciones, sino también 'agentes' sintáctico-semánticos" (Fabbri, op.cit.: 48), involucrados en una determinada dinámica de acciones y valores e inscribiendo todo ello en cronotopos o articulaciones discursivas espacio-temporales específicas. Las narrativas escalares del cambio climático dan cuenta de la transformación de estas configuraciones de sentido, según se cambia de escenario espacio-temporal y la competencia de los agentes requeridos para la acción.

Por otra parte, continuando con el proceso generativo del sentido, con el tránsito de las estructuras narrativas a las discursivas, la tematización del riesgo medioambiental permite "diversas formulaciones de un mismo valor" (Greimas y Courtés, 1982: 405), de tal forma que la naturaleza amenazada puede ser figurada bien como una "defensa" a ultranza de ciertos enclaves privilegiados e incontaminados (reservas naturales), exentos de actividad humana, bien como la reivindicación de ciertas "reparaciones" de los daños infligidos a determinadas comunidades y áreas del planeta por el modelo de desarrollo practicado (justicia redistributiva), o bien como la preservación de un legado intergeneracional (sostenibilidad). Al poner el foco en la tematización, en el componente semántico de las estructuras discursivas del relato informativo del riesgo medioambiental, anterior a su textualización, estas estrategias discursivas pueden ser rastreadas en su funcionamiento transmedial e identificar cómo ciertas significaciones, resultantes del encuentro de una forma expresiva y una forma del contenido, circulan y son compartidas por una sociedad. 
El discurso del riesgo no habla tanto de la naturaleza en peligro como del sentido común proyectado intersubjetivamente sobre aquella parte de la naturaleza cuyo valor interesa proteger y conservar, en un espacio social e histórico determinado. La naturaleza amenazada es la metáfora sobre la que el discurso del riesgo construye y gestiona el sentido y los valores e intereses concurrentes asociados a la construcción social, tensa y conflictiva, del medio ambiente. Así, en la definición del riesgo, el disenso no sólo se refiere a la confrontación de ideas, valores e intereses en juego, sino a la posibilidad de deliberación sobre aquello que es dado de antemano, de lo sensible de una situación sobre la que los ciudadanos perciben, piensan y aprecian o por el contrario, ignoran y desdeñan, de un fenómeno medioambiental. Se trata de observar, en definitiva, la forma en que el discurso del riesgo dibuja los límites de lo que se considera medio ambiente y cómo sus posibles configuraciones sociales y culturales pueden ser pensadas, indagadas, nombradas o argumentadas. En este contexto, frente al disenso como espacio potencial para la deliberación política, la urgencia con que la prensa dramatiza la falta de consenso frente a los peores escenarios del riesgo, considerados ciertos e inminentes, "representa la desestimación de la política como una configuración polémica del mundo común" (Rancière, 2004: 35). El consenso como sentido común impuesto, del que se ha evacuado preventivamente el disenso, representa la imposición de un orden post-político que desplaza la política en favor de la administración experta (Mouffe, 2005), preservándola de la politización de las reivindicaciones particulares.

\subsection{LA SOCIEDAD DEL RIESGO GLOBAL}

La sociedad del riesgo global es uno de los síntomas de la modernidad "reflexiva" (Beck, Giddens y Lash, 1994; Beck, 2002; Giddens, 2010), basada en el saber, cálculo y gestión técnica del riesgo, con capacidad para transformar la configuración del poder a través de la imposición de centros de gerencia y decisión informales (expertos, especialistas, técnicos), con el objetivo de incorporar al mercado aquellos aspectos del mundo, como el medio ambiente, que todavía eran considerados externalidades (prevención, mitigación, adaptación al cambio climático). Como resultado de este proceso, los autores advierten de la progresiva implantación de sub-políticas protegidas del control público y democrático, cuya gerencia es puesta en manos de paneles científicos y centros de asesoramiento tecnológico. En este sentido, la sociedad del riesgo es una sociedad paradójica ya que, a la vez que anticipa una catástrofe global que requerirá acciones cooperadas, reclama la confianza en agentes externos en los que delegar la gestión de problemas que no han sido colectivamente pensados ni evaluados.

Por otra parte, las afirmaciones sobre riesgos no son meras afirmaciones sobre hechos. Los riesgos del cambio climático global son inespecíficos e inconmensurables a escala local, por lo que las conjeturas de causalidad representan, para el público lego, un conocimiento teórico sancionado como verdadero, apodíctico y seguro, que reclama confianza. Así, el discurso del riesgo puede ser observado como una técnica de coacción basada en la producción de expectativas de peligro que propor- 
cionan consenso y estabilidad, junto con los umbrales de daño tolerables en un momento determinado. La información de un determinado riesgo distrae de otros, por lo que "el grado de vigilancia preceptiva y el nivel de aceptabilidad de determinados riesgos proporciona una aproximación a la forma de organización social, a los valores compartidos y a los principios morales que los legitiman" (Douglas, 1996: 92). Por lo general, los desastres denominados "naturales" propician una visión integradora de las desigualdades sociales, aunque generalmente afectan en mayor medida los más desfavorecidos. Las narrativas transmedia del riesgo global, tanto informativas como ficcionales, se han hecho eco de esta temática enunciada como una naturaleza salvaje, herida, en peligro de extinción y con capacidad de afectación global, ante la que se movilizan los sentimientos de solidaridad y de cooperación, por encima de los diferentes grados de responsabilidad imputables por los daños infligidos, no sólo a la naturaleza, sino también a otros colectivos sociales.

\subsection{EL DISPOSITIVO ESPACIO-TEMPORAL DEL RIESGO}

En términos discursivos, el discurso del riesgo se presenta como una "profecía autocumplida" (Merton, 1968:477) pues la definición y expectativa del riesgo da carta de naturaleza a lo que antes sólo era virtual, esto es, el efecto potencial de un desencadenante que todavía no ha llegado a materializarse. El discurso del riesgo medioambiental construye escenarios del infortunio sobre los que se proyecta la incertidumbre producida por la expectativa de consecuencias adversas atribuidas a fenómenos y transformaciones percibidas en el entorno, en los cuales resulta difícil discernir sentido evidente alguno. De esta forma, el discurso del riesgo se perfila como un proyecto de sentido construido sobre el sustrato de la experiencia difusa, fragmentada e informe de lo que se percibe como el caos de los desórdenes medioambientales, cuyas causas y tratamiento se propone elaborar.

Pero la arbitrariedad social, económica y cultural en la selección de valores y bienes a preservar se halla en la base del disenso en la asunción de riesgos. La expectativa de disenso es también la razón por la que el discurso del riesgo se plantea como un discurso polémico, como un campo de maniobras semióticas, un dispositivo estratégico de defensas tácticas frente a posibles refutaciones, destinado a dirimir las tensiones y la violencia ejercida en la definición de lo que diferentes agentes y colectivos consideran qué es un problema medioambiental. Expresiones como "cambio climático", "calentamiento global", "desarrollo sostenible" o "planeta común", frecuentemente esgrimidas por científicos, especialistas y políticos, no hacen sino poner en discurso las tensiones y conflictos que suscitan los desórdenes medioambientales y la pugna por el sentido de cuestiones que no afectan únicamente al entorno natural, sino que son el síntoma insidioso del disenso y de la conflictividad social (Riechman, 1995: 18).

La información del riesgo medioambiental se caracterizan por la disposición de un programa narrativo que se hace cargo de la representación de series de acciones y eventos, significativamente interconectados en un desarrollo a la vez temporal y causal, conformando un argumento explicativo, clausurado y coherente de su obje- 
to. Esta lógica argumental comprende opciones y elecciones de tipo ontológico y epistemológico, gracias a las cuales, el relato informativo de los riesgos medioambientales representa fenómenos complejos como un encadenamiento asequible de acontecimientos, fácilmente comprensibles por el público, pero a costa de alterar las condiciones de pertinencia de los discursos científicos de referencia.

El relato informativo procede también mediante generalizaciones con el fin de transmitir convenciones reconocibles como imaginarios y contextos en los que enmarcar y restringir el universo potencial de valores atribuibles a la noción de medio ambiente. Estos encuadres no son sólo temáticos y cognitivos, sino que prefiguran además el modelo y la competencia del interlocutor con el que el discurso del riesgo se propone dialogar y a su vez movilizar a través del intercambio comunicativo.

Desde el punto de vista del dispositivo temporal, el discurso informativo del riesgo presenta una temporalidad truncada, in medias res, según la cual el pasado y el presente son apenas construidos como el pretexto de una expectativa de futuro inmediato. En el caso de los impactos medioambientales ese futuro prefigurado se torna inminente, inexorable y catastrófico, salvo que se adopten medidas urgentes y solidarias, por encima de diferencias regionales, de clase o de responsabilidad por el deterioro causado.

En el esbozo de los riesgos medioambientales el presente es sistemáticamente sometido a suspense, tensionado por la urgencia de actuaciones inmediatas, sin tiempo para el análisis y valoración de las causas complejas y de los argumentos polémicos a través de los cuales se seleccionan los factores de riesgo, proporcionando relevancia a ciertos fenómenos y criterios en detrimento de otros. De esta forma, los posibles efectos y escenarios del calentamiento global (sequía, inundaciones, vulnerabilidad y dependencia) pueden ser interpretados por los ciudadanos en función tanto de la expectativa de un gran daño, como de la oportunidad para el beneficio propio (comercio, reconstrucción e influencia). El dispositivo temporal caracterizado por la inminencia de las catástrofes anunciadas coopera así con la refutación de voces alternativas que pudieran poner en cuestión los presupuestos sobre los que se han erigido los escenarios de riesgo, seleccionados como pertinentes para la empresa común y para el reparto desigual de competencias para la acción.

Solidariamente con el dispositivo temporal, el dispositivo espacial plantea diferentes marcos territoriales que dan lugar a narrativas escalares en función del ámbito de actuación seleccionado y de los actores convocados para su desempeño. Así, mientras a los problemas globales corresponden acciones comunes referidas a un sujeto abstracto -la humanidad- despojado de interés político o económico, en las que el ciudadano no puede participar sino como un agregado solidario, en el ámbito local de actuación se pone el énfasis en las acciones individuales, domésticas, anónimas y cotidianas, desafectadas del campo de participación, de decisión y de acción política y comunitaria.

Finalmente, el dispositivo actoral del discurso del riesgo privilegia ciertos protagonistas (científicos, políticos, técnicos y gestores medioambientales, ecologistas) como voces a las que se reconoce distinto grado de pertinencia en la definición de los problemas medioambientales en función del ámbito escalar de actuación, así como la legitimidad, la competencia y la capacidad de actuación frente a las ame- 
nazas que pesan sobre el entorno. Estas voces son las responsables de extraer de lo inefable, de la caótica manifestación de los cambios percibidos en el acontecer y según la escala espacial en la que actúan, algún sentido articulado mediante la puesta en relación de causas y efectos, de desencadenantes y consecuencias indeseadas, en situaciones que es preciso "gestionar eficientemente", "erradicar", "obviar" o en su caso, interpretar en términos de equidad y de justicia social (Lorente, Antolín y Doblas, 2009).

\section{LAS NARRATIVAS ESCALARES DEL RIESGO MEDIOAMBIENTAL}

En el contexto del nuevo paradigma de un capitalismo líquido (Bauman, 2007), en el que las escalas espaciales son constantemente redefinidas, las narrativas escalares son relatos acerca de los cambios en la configuración espacial de los procesos sociopolíticos (González, 2005:10). Estas narrativas disponen cronotopos específicos en los que se perfilan actores comprometidos con un repertorio restringido de posibilidades de deliberación, de participación y de acción política (Swyngedouw, 2011: 23).

En virtud de las narrativas escalares, la globalización de los problemas y tensiones medioambientales puede ser informada como la necesidad de una gobernanza transnacional y multilateral de los mismos, o bien como una oportunidad para aprovechar la capacidad de determinados países para plantear y tratar de imponer compromisos inalcanzables para otros. De esta forma, se trata de utilizar la posición de influencia y de desarrollo tecnológico para crear nuevas opciones de mercado en torno al reparto de cargas y beneficios asociados a la medida y control de las amenazas medioambientales, así como para la gestión, no sólo técnica, sino también política y económica, de los umbrales de aceptabilidad de los mismos.

\subsection{LA LÓGICA DEL CONSENSO EN LA ESCALA GLOBAL}

El consenso general en la definición y alcance de los riesgos medioambientales además de delimitar lo pertinente y de excluir lo impertinente para la articulación de las posibles políticas de paliación, significa ante todo la exclusión del debate sobre lo que es dado de antemano, de aquello que ha sido seleccionado, evaluado e impuesto no sin fuerza y violencia, siquiera simbólica, y que por tal razón ha adquirido carta de naturaleza.

La prensa española, en la misma línea que la internacional, se refería a la última conferencia del clima, celebrada en Doha (Qatar, 2012), como un "fracaso anunciado", debido al contexto de crisis económica y de repunte del escepticismo acerca del proceso de calentamiento global. Y con el propósito de buscar consensos y acuerdos "realistas", periodistas, investigadores y académicos parecían coincidir en la necesidad de que fueran los mayores productores de gases de efecto invernadero los que concertaran las condiciones de una pretendida paz forzada, con la que se pusiera fin al disenso y a la falta de compromisos vinculantes para alcanzar los objetivos de Kioto o redefinirlos para acuerdos posteriores. Pero, si bien los efectos de las emi- 
siones de los gases de efecto invernadero tienen una capacidad de afectación global, ni su generación ni sus impactos negativos sobre el medio ambiente, sobre el desarrollo y sobre las economías de los países participantes en la conferencia se reparten de forma homogénea.

Las disputas por el sentido del medio ambiente y la definición del umbral de deterioro tolerable han formado parte del juego político escenificado en estos foros internacionales. Ahora bien, si la expectativa de un amplio consenso en cuya deliberación participaran todas las partes caracterizaba la hoja de ruta de estas convocatorias, a partir del fracaso de la conferencia de Copenhague tal expectativa ha sido restringida a la consecución de acuerdos posibilistas entre aquellas partes que paradójicamente tienen el mayor potencial contaminante y mayores intereses en su control, pero minimizando sus efectos no sobre el medio ambiente, sino sobre sus propios sistemas productivos y estrategias de desarrollo.

Una vez excluidos los países emergentes y el disenso de la deliberación política sobre el control de las emisiones, la búsqueda realista de soluciones consistiría en un acuerdo de mínimos sobre cuestiones relativas al control técnico y a la financiación de la adaptación al cambio climático, postergando para un futuro más o menos lejano el objetivo de reducción de las emisiones. De esta forma, la gobernanza global de un problema global se transforma, mediante el discurso del riesgo, en una "función meramente gerencial, privada de su auténtica función política" (Zizek, 2008), salvando así el escollo del consenso compartido al que comprometía el reglamento marco de Naciones Unidas.

La prensa nacional de referencia ha abordado estos escenarios informativos dibujando un panorama confuso de luces y sombras sobre los procesos de negociación política en torno a los principales compromisos relacionados con el protocolo de Kioto. Tras el fracaso de Copenhague, la prensa recogía las deliberaciones de la conferencia de Cancún con titulares como "La UE propone un 'Kioto2' para salvar la Cumbre del Clima” (Vidal, El Mundo, 10/XII/2010) o “¿Puede la ONU gobernar el clima?... una cumbre bloqueada por desacuerdos" (Sabartés, La Vanguardia, 10/XII/2010) poniendo el acento en la falta de capacidad para el consenso y gobernanza del cambio climático y en la dramática situación en que se encuentran las negociaciones "Cuenta atrás para aprobar una prórroga de Kioto" (Acosta, $A B C$, 04/XII/2012).

La necesidad de adaptación al cambio climático, uno de los temas más controvertidos de las negociaciones, ha sido argumentado como el sometimiento no sólo a las condiciones resultantes de un proceso natural, sino a las consecuencias medioambientales de las decisiones tomadas por un puñado de países acerca de los ritmos de afrontamiento del cambio climático, salvaguardando los intereses que subyacen a tales compromisos. $\mathrm{Y}$ en este contexto interpretativo, al mismo tiempo que se habla de ayuda a la adaptación para los países en vías de desarrollo, aparentemente incapacitados para gestionar el riesgo definido y generado por otros, se expresa en términos de iniciativa y de capacidad de control la actuación de los países desarrollados. Todo ello redunda en la representación de un reparto desigual de competencias y de capacidad de acción, sustentada por el relato informativo de las deliberaciones sobre el riesgo medioambiental. De esta forma, si bien en la información de las negociaciones sobre el cambio climático y su gobernanza global se reconoce implí- 
citamente el disenso y los diferentes valores e intereses en juego, se somete todo ello a una dramaturgia que conjuga la discrepancia como la resistencia irracional y el seguro abocamiento hacia un desenlace fatal.

\subsection{LA ESTRATEGIA DE LA OPORTUNIDAD EN LA ESCALA NACIONAL}

Ahora bien, cuando la prensa de referencia se hace eco de la participación española en estos encuentros internacionales, las narrativas escalares presentan un dispositivo enunciativo diferente. Por lo general, como advierten algunos titulares, España aparece enmarcada en la ambiciosa estrategia europea de reducción de emisiones "La UE promueve un texto que considera insuficiente el pacto de Copenhague" (Sabartés, La Vanguardia, 11/XII/2010), pero sin perder de vista las oportunidades particulares que brinda la imposición de un consenso y gerencia globales: "España, satisfecha con el consenso" (Agencias, El Mundo, 12/XII/2010).

El relato informativo del protagonismo reconocido a la UE y a la implicación española en la escena global del cambio climático sigue, en términos generales, el argumento de la "oportunidad" como lógica dominante del consenso a alcanzar. La oportunidad, desde el punto de vista de los países de la UE en relación con la problemática medioambiental, significa ante todo la transformación del compromiso ético y solidario para abordar soluciones comunes ante las amenazas globales, en un aprovechamiento de las ventajas y de los recursos disponibles para alcanzar posiciones de liderazgo y de influencia en el mercado. Desde que el denominado "Informe Stern" (Stern, 2007) se convirtiera en referencia del interés económico de la prevención y paliación de los efectos del cambio climático (Giddens, 2010), el discurso del riesgo medioambiental establece, más o menos explícitamente, la intimidad de la ética y del mercado, a través de la premisa de la oportunidad. Una oportunidad que, junto con el relato del liderazgo en la limitación de las emisiones promovida por la UE, versa principalmente acerca de las medidas a tomar en materia de producción y consumo energético para el crecimiento de las respectivas economías.

En la escala nacional, el discurso del riesgo pone el énfasis en la medida de las causas e impactos, pero a costa de ignorar tanto los aspectos no mensurables de los desórdenes ambientales, así como la externalización de los mismos por parte de aquellos países con capacidad para gestionar su efectos no deseados. De esta forma, el discurso del riesgo contribuye a la economía política que hace de los problemas medioambientales una oportunidad de crecimiento para los países desarrollados, en tanto que condiciona las posibilidades de autonomía y modernización de los países sometidos a la tutela medioambiental de los primeros.

Las narrativas de escala nacional hacen hincapié en la idea de que el principio de prudencia manejado en la escala internacional, consistente en el precepto de que "más vale prevenir que curar", puede conducir a la inacción debido a que esta perspectiva se centra en un solo aspecto del riesgo, la posibilidad de sufrir daños, mientras descuida otro de sus dos posibles sentidos: el de la oportunidad basada en el cálculo de la probabilidad y en el análisis del coste-beneficio en un escenario calculado de riesgo. En este contexto interpretativo, la lógica de la oportunidad reclama una con- 
vergencia política y económica como estrategia frente al cambio climático. Una convergencia que versa sobre la armonización de los preparativos frente a los peligros con el aprovechamiento de las oportunidades, haciendo coincidir a escala nacional los valores y objetivos multilaterales frente al cambio climático global con la innovación y la eficiencia tecnológica, de tal forma que se generen nuevos mercados y ventajas competitivas para quienes la impulsan. Pero esta convergencia no apunta necesariamente, por si misma, al compromiso con el imperativo de desarrollo de los países pobres, ni a una mayor cohesión y justicia social, sino más bien al ahondamiento de la brecha que separa a quienes tienen los recursos para la evaluación y el control de los riesgos y aquellos países y grupos sociales que carecen de ellos y de la posibilidad de aprovechamiento de las oportunidades que representan.

En este sentido, las narrativas escalares del riesgo funcionan como fábulas aleccionadoras con capacidad para reorientar el sentido y transformar los costes medioambientales que antes se consideraban externalidades, en internalidades incorporadas al mercado. De ahí también que, para algunos estados europeos que lideran la búsqueda de acuerdos maximalistas para la gobernanza global del riesgo medioambiental, el interés reside más en el aprovechamiento de la oportunidad que representan los nuevos mercados de compensación (fiscalidad del carbono) y en la dependencia de los países en vías de desarrollo para acceder a tecnologías energéticas eficientes, que en la reducción efectiva de los factores de riesgo. Este es el doble precio que los países pobres tienen que pagar en aras del "beneficio común". El precio político de participar en un escenario en el que cuentan con escasas posibilidades de decisión y el precio económico de acceso a unos recursos naturales escasos, cuya alternativa son fuentes de energía eficientes por las que han de pagar el coste de la transferencia tecnológica. Todo ello en un contexto de análisis estratégico de la vulnerabilidad y resistencia de los países y de los grupos sociales no sólo frente a las consecuencias negativas del calentamiento global, sino frente a las políticas destinadas a combatirlo, definidas por un grupo reducido de países guiados por la lógica del interés y la oportunidad, en un mercado global concurrente de amenazas y oportunidades.

\subsection{LA RACIONALIDAD TÉCNICA EN LA ESCALA LOCAL}

La narrativa de la actualidad medioambiental en el ámbito local presenta espacios de sentido y de participación desasidos del escenario anterior. La información local se interesa por el tratamiento de los acontecimientos globales con repercusión potencial en el espacio próximo al lector. Sin embargo, los riesgos medioambientales son complejos e inespecíficos, por lo que la prensa local tiende a presentar los escenarios de riesgo más negativos, independientemente de su cálculo y probabilidad en el ámbito concreto de influencia del medio informativo, pero identificando en este los síntomas inequívocos del deterioro medioambiental global. De esta forma, acontecimientos que se producen en el entorno próximo pero que tienen un alcance reducido y excepcional son presentados como la confirmación de los pronósticos más aciagos (Gardner, 2008: 336). 
Por otra parte, si bien es el ámbito local de participación el más cercano y accesible al ciudadano, los medios de comunicación dedican escasa atención a los mecanismos dispuestos para ello. De esta forma, la relación entre las actuaciones particulares y sus efectos y consecuencias medioambientales globales, así como entre el ámbito local de participación y los escenarios globales de gobernanza y decisión, queda difuminada y mediatizada por el relato informativo de la inmediatez e inexorabilidad de la catástrofe, en un contexto de escepticismo ante la falta de legitimidad política para su gobierno.

En la escala local, muchas de las estructuras participativas creadas a tal fin (agendas 21 locales, consejos de transporte, urbanismo, medio ambiente) trataron de dar respuesta a la necesaria adecuación de la gestión administrativa a las nuevas formas de participación ciudadana. Sin embargo, tras su constitución, estas estructuras han reorientado sus objetivos hacia la recogida de información sobre la diversidad de intereses en concurrencia con el fin de anticipar y gestionar posibles conflictos, en detrimento de la construcción de un nuevo sujeto político, confundiendo la información y la consulta a los ciudadanos con la capacidad de decisión y acción. A este respecto, mientras la administración local define, construye y planifica la realidad social sobre la que se propone intervenir (Beltrán, 1991), esta se manifiesta de forma compleja, constituida por dinámicas cambiantes, cuyas transformaciones desbordan el marco de intervención previsto. Este es uno de los aspectos más comprometidos de la gestión del disenso en el momento de decidir la ubicación de servicios o infraestructuras con impactos medioambientales (vertederos de residuos, plantas de reciclaje), cuyo grado de afectación se distribuye de forma desigual entre la población, pero cuyo interés es reclamado e informado tanto por la administración, como por los medios de comunicación, en términos de bien común.

En estos escenarios de disenso, los sistemas de racionalidad que orientan la intervención de las administraciones pueden apelar a la aplicación de normas y al respeto de la legalidad vigente en materia medioambiental o a la negociación y a la búsqueda de consenso, propiciando la participación de diferentes agentes sociales en la deliberación de las diversas perspectivas y estrategias posibles de intervención. Pero mientras la lógica normativa trata de asegurar la administración de una realidad previamente definida, encapsulando el riesgo como si de un sistema cerrado y estático se tratara, la negociación requiere el reconocimiento de una realidad tensa y compleja, en constante transformación. Y en el momento en que la deliberación y el disenso amenazan con desbordar el marco normativo establecido se crean estructuras técnicas, especializadas, bajo el argumento del sostenimiento y mejora de la eficiencia funcional. Como resultado de este bucle al que la dinámica administrativa se ve abocada, surgen estructuras para-administrativas, no sometidas a sus reglas y procedimientos, pero con capacidad para definir una nueva realidad en términos de racionalidad técnica. Esta realidad se basa en un nuevo tipo de consenso experto, exento de deliberación política, guiado por objetivos de tipo estratégico y competitivo.

Los medios de comunicación locales participan frecuentemente de esta lógica técnico-administrativa haciendo hincapié en la extremada complejidad de los problemas medioambientales. Para ello, la información de actualidad exhibe los diferentes puntos de vista, subraya los posicionamientos irreconciliables y el cruce inextricable de 
acusaciones mutuas entre las partes implicadas en los litigios medioambientales, al tiempo que abre la puerta a la definición experta de un nuevo espacio de sentido y decisión. Los ciudadanos son insistentemente informados de su creación (ventanillas de consulta, de apelación y reclamación) y consultados acerca de su valoración (encuestas de opinión), en tanto que consumidores de "servicios" medioambientales. Sin embargo, quedan sistemáticamente excluidos de la definición y discusión de los problemas que les afectan, todo ello en beneficio del rigor y eficacia del saber especializado.

El sujeto emergente en el escenario del disenso y del debate político cede así su competencia a un nuevo sujeto, el experto, y a la racionalidad técnica privilegiada para afrontar los problemas medioambientales. Al mismo tiempo, el ciudadano pasa a ser usuario o consumidor de servicios y recursos ambientales previamente programados, sobre cuyas prestaciones y grado de satisfacción es insistentemente informado y consultado, pero de cuya deliberación política es sustraído con el argumento, no ya de una mayor cohesión social, sino de una mayor eficiencia en su gestión. Como resultado de esta dinámica, aquel nuevo sujeto político con capacidad de decisión y de acción al que aspiraban tanto las reformas administrativas, como la movilización informativa en torno a los problemas medioambientales, se transforma en el producto de una estrategia de marketing, en la lógica de la racionalidad técnica aplicada a conflictos de cuya definición y gestión la ciudadanía ha sido preventivamente desplazada.

\section{CONCLUSIONES Y DISCUSIÓN}

Cuanto más complejo y vasto es un problema mayor dificultad comporta su definición, así como la articulación de mecanismos de información y participación en su resolución. Consecuentemente, las narraciones informativas implicadas en la difusión del conocimiento científico y de la deliberación de las estrategias para el tratamiento de los riesgos medioambientales, requieren el reconocimiento de la diversidad de escenarios, expectativas e intereses que participan en la definición y gestión de los mismos. Para ello, es preciso también que los diversos agentes implicados en las diferentes escalas de afectación y gestión reconozcan su competencia para definir, debatir y acordar las formas de participación en las decisiones que les afectan, así como sus repercusiones y grado de afectación para los demás. De esta forma, los problemas y riesgos medioambientales pueden ser re-politizados y pensados no solamente en términos de naturaleza afectada, sino también en función de los umbrales socialmente acordados de naturalezas y de formas de vida a conservar o, en su caso, sacrificar.

La información de la actualidad medioambiental incorpora narrativas escalares en las que se inscribe la definición y cursos de acción frente a los riesgos medioambientales. En estos escenarios narrativos se dirime el disenso y se identifican los agentes y las formas de participación privilegiadas en función del bien común, de la oportunidad o de la racionalidad técnica, según los casos.

La gestión informativa de la incertidumbre asociada a los riesgos medioambientales en el ámbito local se concreta en unas narrativas que enmarcan los fenómenos 
naturales próximos al ciudadano en los peores pronósticos. Estos escenarios catastróficos sirven de pretexto para referir al saber técnico y experto la definición y solución de problemas susceptibles de deliberación política, pero ante los que se reclaman acciones comunes, libres de disenso, frente a daños que no tienen precisamente un reparto ni común, ni igualitario, de costes y beneficios. El tratamiento catastrofista, inmediato y de afectación global de los riesgos medioambientales, lejos de animar la participación ciudadana en la deliberación y gestión de sus potenciales efectos, propicia el escepticismo y la delegación a la gerencia tecno-científica la resolución post-política de las estrategias de afrontamiento.

\section{BIBLIOGRAFÍA}

Bauman, Z. (2007): Tiempos líquidos. Vivir en incertidumbre. Barcelona: Tusquets.

BECK, U. (2002): La sociedad del riesgo global. Madrid: Siglo XXI.

BECK, U. (2010): "Climate for Change, or How to créate a Green Modernity?", Theory, Culture \& Society, 27. March, pp. 254-266.

BECK, U., GIDDENS, A., LASH, S. (1994): Reflexive modernization: politics, tradition and aesthetics in the modern social order. Stanford: Stanford University Press.

BELTRÁn, M. (1991): La realidad social. Madrid: Tecnos.

Boykoff, Maxwell T. (2011): Who Speaks for Climate? Cambridge University Press.

DíAz Nosty, B. (2009): "Cambio climático, consenso científico y construcción mediática. Los paradigmas de la comunicación para la sostenibilidad." Revista Latina de Comunicación Social, 64. Págs. 99-119 (DOI: 10.4185/RLCS-64-2009-808-99-119).

Douglas, M. (1996): Aceptabilidad del riesgo en ciencias sociales. Barcelona: Paidós.

FABBRI, P. (1995): Tácticas de los signos. Barcelona: Gedisa.

-, (2004): El giro semiótico. Barcelona: Gedisa.

GARDNer, D. (2008): Risk: The Science and Politics of Fear. London: Virgin.

GidDENS, A. (2010): La politica del cambio climático. Madrid: Alianza Editorial.

GonZÁLEZ, S. (2005): "La geografía escalar del capitalismo actual”, Scripta Nova. Vol. IX, num. 189, <http://www.ub.es/geocrit/sn/sn-189.htm>, consultado el 4/XII/2009.

Greimas, A. J.y CourTÉs, J. (1982): Diccionario razonado de la teoría del lenguaje. Madrid: Gredos.

IMBERT, G. (2008): El transformismo televisivo. Madrid: Cátedra.

LoRente, J.I., Antolín, J.E., Doblas, F.J. (2009): "La construcción mediática de lo ecológico. Estrategias discursivas de la información de actualidad", Revista Latina de Comunicación Social, 64, pp. 315-327 (DOI: 10.4185/RLCS-64-2009-825-315-327).

Lorenzoni, I. y Pidgeon, N. (2006): Defining the Dangers of Climate Change and Individual Behaviour. Norwich: Centre for Environmental Risk, University East Anglia.

Lowe, Th., Brown,K., Dessai, S., França, M., Haynes, K., Vincent, K. (2006): "Does tomorrow ever come? Disaster narrative and public perceptions of climate change", Public Understanding of Science, 15, octubre, pp. 435-457.

Merton, R.K. (1968): Social Theory and Social Structure. N. York: The Free Press.

NorgaArd, K.M. (2011): Living in Denial. Climate Change, Emotions, and Everyday Life. Cambridge: MIT Press.

Pettenger, E. (2007): The social construction of climate change. London: Ashgate. 
RAJendRA, K., PACHAURI, Resinger, A. (Eds.) (2007): "IPCC Intergovernmental Panel on Climate Change Climate Change 2007: the AR4 Synthesis Report". $<$ http://www.ipcc.ch/home_languages_main_spanish.shtml\#.T3Qvdo7rbXQ>, 7/5/2008

RANCIÈre, J. (2004): Aux bords du politique. París: Gallimard.

RAnCIÈre, J. (2006): The Politics of the Aesthetics. London: Continuum.

Ricoeur, P. (2008) Tiempo y narración II. Buenos Aires: Siglo XXI.

RiEchmanN, J. (1995): “Desarrollo sostenible: la lucha por la interpretación”, en VV.AA. De la economía a la ecología. Madrid: Trotta.

SLImak, M.W., Dietz, T. (2006): "Personal values, beliefs and ecological risk perception", Rysk Analysis, n. ${ }^{\circ}$ 26, pp.211-220.

STERN, N. (2007): Informe Stern. La verdad del cambio climático. Barcelona: Paidós.

SwYNGEDOUw, E. (2011): “¡La naturaleza no existe! La sostenibilidad como síntoma de una planificación despolitizada", Urban, NS01, pp. 41-66.

ZIZEK, S. (2008): "Censorship Today: Violence or Ecology as a New Opium for the Masses", Resources for dilletantes, en <http://fordiletante.wordpress.com/2008/05/07 $\geq$

\title{
RESUMEN
}

Este trabajo aborda un análisis de las estrategias discursivas implicadas en el tratamiento de la información de actualidad del riesgo medioambiental, publicada en la prensa española de mayor difusión durante las últimas conferencias internacionales sobre Cambio Climático (UNFCCC), relativas a la renovación del protocolo de Kioto. Como resultado, se discriminan tres estrategias narrativas escalares a través de las cuales se gestionan las tensiones implicadas en la definición del riesgo medioambiental, con proyección en el ámbito internacional, nacional y local, respectivamente.

Palabras clave: comunicación, discurso, prensa, riesgo, medio ambiente.

\section{The informative managent of enviromental risk}

\begin{abstract}
This work tackles an analysis of the discursive strategies involved in the treatment of the current affairs information published in the Spanish newspapers of wider diffusion. The focus of this analysis is set on the topic of environmental risk as framed by the context given by the recent UN Conferences on Climate Change, where the main issue was the renewal of the Kyoto Protocol. The result includes the identification of three narrative strategies that manage the tensions and conflicts involved in the definition of the environmental risk and projected on international, national and local scopes.
\end{abstract}

Keywords: communication, discourse, newspaper, risk, environment. 